\title{
Nonlinear and hysteretic modelling of magnetorheological elastomer base isolator using adaptive neuro-fuzzy inference system
}

\author{
Yang $\mathrm{Yu}^{1, \mathrm{a}^{*}}$, Yancheng $\mathrm{Li}^{1, \mathrm{~b}}$, Jianchun $\mathrm{Li}^{1, \mathrm{c}}$, Xiaoyu $\mathrm{Gu}^{1, \mathrm{~d}}$, Sayed Royel ${ }^{1, \mathrm{e}}$ and \\ Arati Pokhrel ${ }^{1, f}$ \\ ${ }^{1}$ Faculty of Engineering and IT, University of Technology Sydney, Sydney, 2007, Australia \\ ayang.yu@uts.edu.au; byancheng.li@uts.edu.au; jjianchun.li@uts.edu.au; \\ dxiaoyu.gu@student.uts.edu.au; 'sayed.royel@uts.edu.au; 'arati.pokhrel@student.uts.edu.au
}

\begin{abstract}
Keywords: Magnetorheological elastomer (MRE) base isolator, hysteresis behaviour,
\end{abstract} nonparametric modelling, adaptive neuro-fuzzy inference system (ANFIS)

\begin{abstract}
Magnetorheological elastomer (MRE) base isolator is a semi-active control device which has currently obtained increasing attention in the field of vibration control of civil structures. However, the inherent nonlinear and hysteretic response of the device is regarded as a challenge aspect for using the smart device to realize the high performance. Therefore, an accurate and robust model is essential to make full use of these unique features for its engineering applications. In this paper, to solve this issue, adaptive neuro-fuzzy inference system (ANFIS) is utilized to characterize the dynamic behavior of the device. In this proposed model, the inputs are historical displacements and applied current of the device while the output is the shear force generated. To validate its forecast performance, the ANFIS model is also compared with some conventional models. Finally, the result verifies that ANFIS has the best perfection ability among existing MRE-based device models.
\end{abstract}

\section{Introduction}

Magnetorheological elastomer (MRE) base isolators have been developed and regarded as one of the most ideal devices for implementation of semi-active control in base isolation system of civil structures against various hazard external excitations such as winds and earthquakes. This newly developed device was designed using MRE smart materials, which are mainly made up of enormous iron additives and gel-like materials. The magnetic particles are mixed within a non-magnetic elastomer matrix, normally a mixture of natural silicon rubber and silicon oil [1]. When the magnetic field is applied to the MRE isolator, the device will show the obvious variation in both shear modulus and damping properties. Nevertheless, one of the major drawbacks is nonlinear and hysteretic relationships between shear force and displacement/velocity of the device. To make full use of this novel device to realize the semi-active control, an accurate and robust model should be developed before the controller is applied into practice.

Recently, several classical hysteresis models were selected to portray nonlinear responses of MRE base isolators, such as Dahl model [2], Bouc-Wen model [3], hyperbolic model [4], LuGre friction model [5] and strain-stiffening model [6-7]. These models are able to provide perfect performance on modelling of MRE isolators. However, the highly nonlinear differential equations in the models can bring the inconvenience for the implementation of feasible control system because of the calculation requirement. Moreover, the performances of these models are heavily dependent on model structures and parameter values. Once the parameter values are inaccurately assigned, the effectiveness of the control system will be affected.

In this work, a novel model based on adaptive neuro-fuzzy interference system (ANFIS) is developed to characterise MRE base isolator. ANFIS adopts a hybrid algorithm which integrates least square method with back propagation gradient descent method to build a fuzzy inference system in which membership functions are updated iteratively based on the known group of input and output samples. The target of ANFIS is to combine the optimal characteristics of neural network and fuzzy system, which has been proved as an ideal function approximation method. In the proposed ANFIS model, input variables are historical displacements and applied current of the device while the output 
variable is shear force generated. Finally, the experimental data measured from a MRE base isolator with various excitations are utilised to assess the performance of the proposed ANFIS model. The results show that it outperforms other MRE models in terms of error-based performance indices between predicted results and practical measurements.

\section{Adaptive MRE base isolator}

Inspired by the distinct features and advantages of MRE material, Li et al. developed a highly adjustable adaptive MRE base isolator transformed from a traditional laminated rubber bearing structure [8]. This design is to solve the drawbacks of the conventional isolator caused by the intrinsic passive property by replacing the common rubber element with new MREs in which shear modulus and damping can be varied with the applied current level. Fig. 1 shows the photo and schematic graph of the MRE isolator prototype. As shown in the cross-section view of the isolator, the novel adaptive isolator has laminated structure with alternative multilayer MRE sheets and thin steel plates. In this design, there are 26 layers of steel sheet with thickness of $1 \mathrm{~mm}$ and 25 layers of MRE sheets with thickness of $1 \mathrm{~mm}$. The diameter of the MRE and steel is $120 \mathrm{~mm}$.

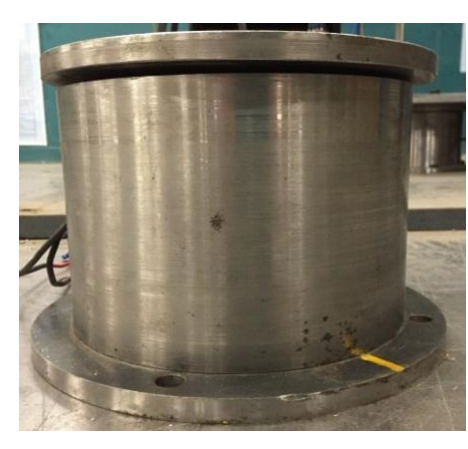

(a) Photo

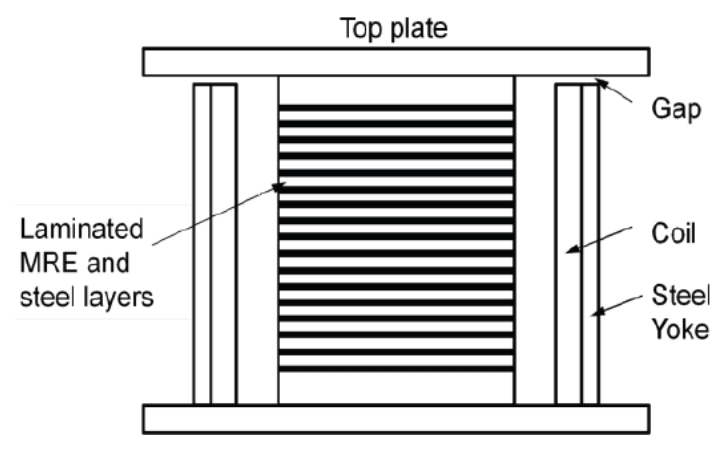

(b) Cross-section view

Fig. 1 Photo and structure of MRE base isolator
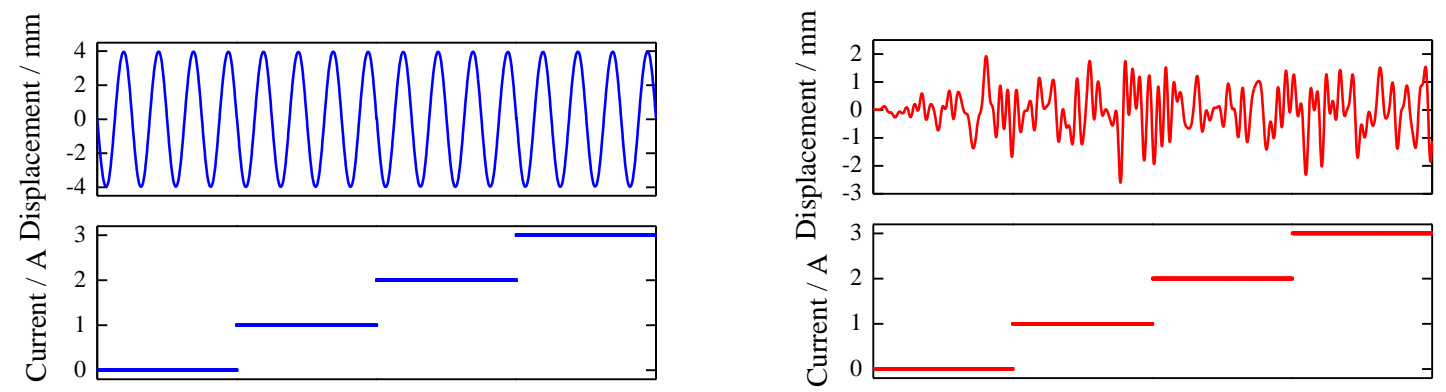

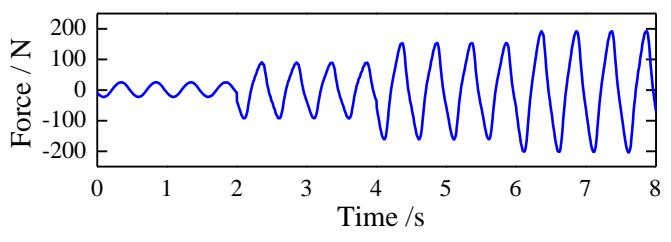

(a) Harmonic excitation

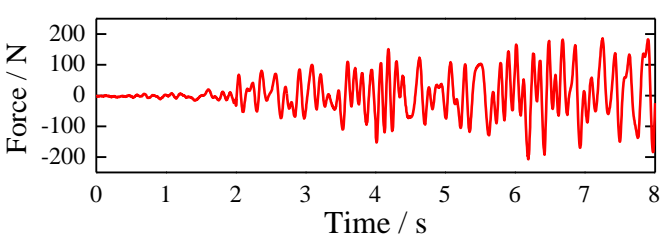

(b) Random excitation

Fig. 2 Displacement, current and force responses of MRE base isolator

In this newly developed device, a solenoid is equipped outside the laminated structure to generate a uniform magnetic field after it is electrified. Under maximum allowed lateral displacement, $15 \mathrm{~mm}$, the vertical load carrying capacity of the MRE isolator is $50 \mathrm{~kg}$ with the absence of magnetic field. The vertical loading capacity increases with the magnetic field and declines with the shear displacement.

For the dynamic testing of MRE base isolator, two types of excitations are used to load the device, including harmonic and random excitations. In the harmonic excitation test, the loading frequency is 
$2 \mathrm{~Hz}$ and amplitude is $4 \mathrm{~mm}$. In the random excitation test, the loading frequency is controlled between $1 \mathrm{~Hz}$ and $20 \mathrm{~Hz}$, and the maximal loading amplitude is $5 \mathrm{~mm}$. For all excitation types, the current levels are varied from 0 A to $3 \mathrm{~A}$ to measure different responses of device corresponding to different magnetic fields. During the test, the sampling rate and duration are selected as $256 \mathrm{~Hz}$ and 8 s, respectively. Fig. 2 shows the displacement, current and shear force responses under different loading conditions. In this work, $50 \%$ of collected data are randomly selected as the training samples for ANFIS model construction while the rest is used as the validation data for model performance assessment.

\section{ANFIS model for MRE base isolator}

The ANFIS has great capacity in dealing with the imprecision and uncertainty of nonlinear systems and is frequently implemented to build the fuzzy IF-THEN rules of logical inference through its powerful and effective data mining techniques [9]. Data mining techniques are used to extract the main features inside the data sets and self-configure their interactive relations. The Sugeno fuzzy model is used to establish IF-THEN rules. Regarding the learning procedure and parameter adjustment, a feedforward neural network coupled with a supervised learning algorithm is implemented, which effectively and appropriately adjusts all parameters of the fuzzy inference system so that the model can possess the self-learning and generalization capacity.

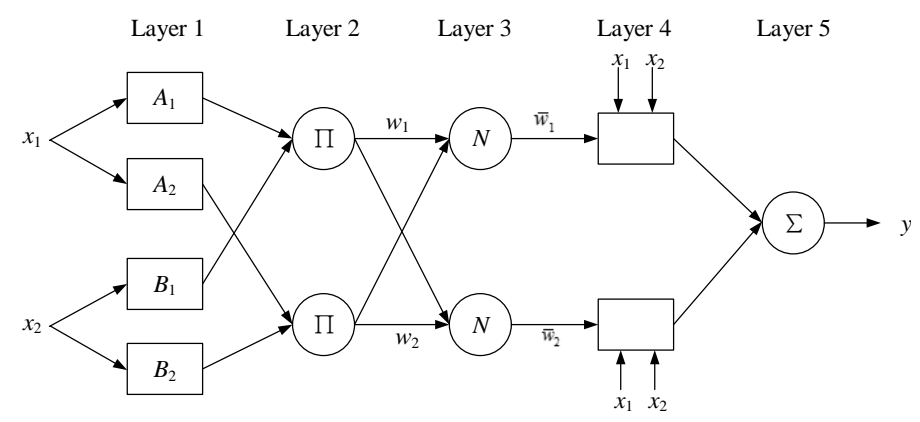

Fig. 3 Structure of ANFIS

Fig. 3 gives the structure of ANFIS, which is regarded as a five-layer neural network. The first layer consists of $n$ membership functions, where the fuzzy decision rule is realised in each function. Each input degree can be obtained in the range of $[0,1]$ in the fuzzification layer. The second layer is rule layer. The output of this layer is the outcome of related degrees from the first layer. The third layer is the normalization layer, which is used to compute the ratio of each ith rule's firing strength to the sum of all rules' firing strength. The fourth layer is the defuzzification layer, where each node computes the contribution of $i$ th rules towards the whole output. The last layer is to summarise all the outputs.

In this work, ANFIS model is considered as an efficient calculation platform which is perfectly suitable to the realisation of nonlinear associations through the construction of many piecewise linear relationships. The requirement to construct such a function is able to obviously reflect the nonlinear dynamic nature of the MRE base isolator. Therefore, the proposed model for MRE base isolator is composed of three inputs and one output, that is displacement at time $t$, displacement at time $t-1$ and current level as input nodes and shear force as output node. This model is based on a first order Takagi-Sugeno-Kang (TSK) architecture that is generally composed of $r$ rules of the form, given as follows:

Rule $i$ : IF $x$ is $A_{i}$ and $\hat{x}$ is $B_{i}$ and $f$ is $C_{i}$, THEN $z_{i}=p_{i} x+q_{i} \hat{x}+s_{i} f+d_{i}$

where $A_{i}, B_{i}$ and $C_{i}$ denote the fuzzy sets; $x, \hat{x}$ and $f$ denote the input variables; $z_{i}$ denotes the output variable; $m_{i}$ denote the output parameters of fuzzy system.

The setup and validation procedure of the proposed ANFIS model can be concluded as following steps:

Step 1. Randomly select training and validation samples obtained from experimental testing; 
Step 2. Build the ANFIS model which is used to express the nonlinear relationships between displacement and current responses and shear forces of MRE base isolator;

Step 3. Assess the model performance based on the error indices between practical results and predictions from the trained model, given the same inputs.

\section{Result and discussion}

Fig. 4 depicts the input membership functions before training and after training for the case of random excitation using ANFIS-4. It is clearly seen that the shapes of membership functions have changed in different stages (initial and final) except that of current input. The similar result can also be obtained in the case of harmonic excitation.

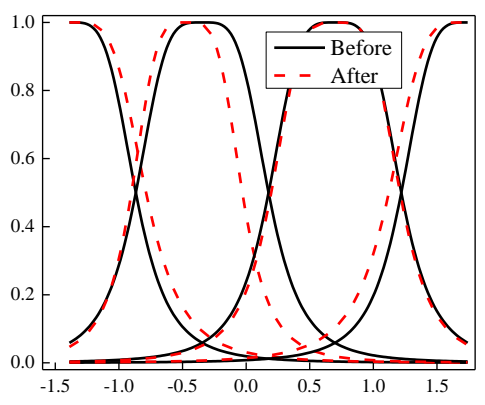

(a) Input 1

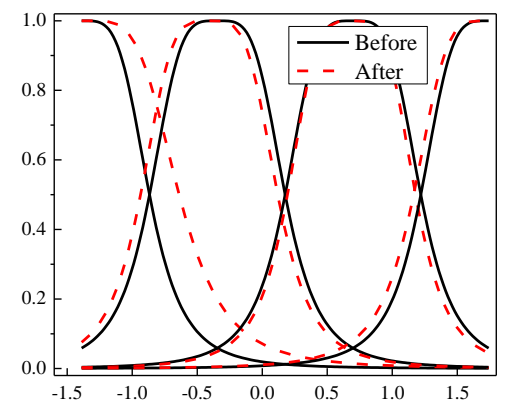

(b) Input 2

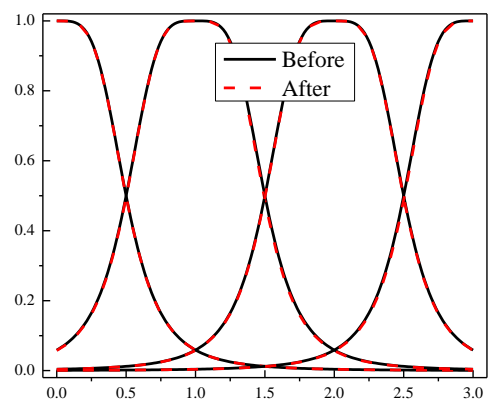

(c) Input 3

Fig. 4. Input membership functions before and after training.

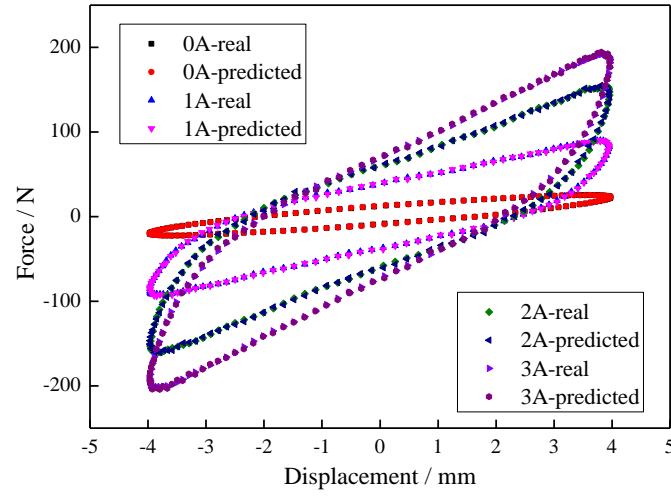

(a) Force-displacement response

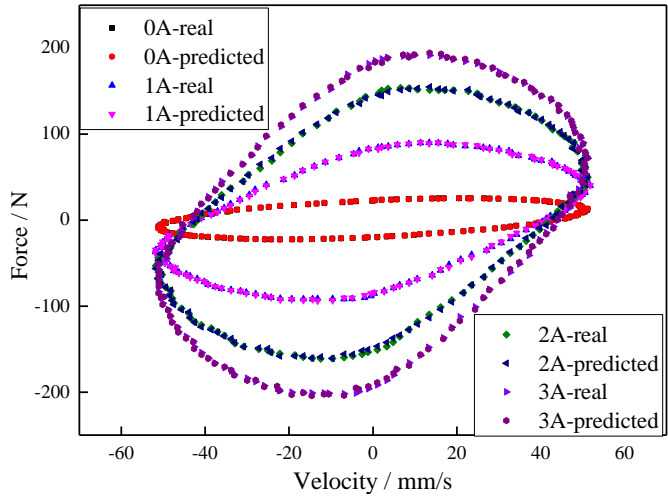

(b) Force-velocity response

Fig. 5 Comparison between experimental and predicted responses

Fig. 5 compares the measured force-displacement/velocity responses with predicted responses from the proposed ANFIS model under harmonic excitations with different applied currents. It can be clearly seen that the predicted hysteresis loop at each current level fits the corresponding experimental data very well, proving the performance of the trained ANFIS model for portraying the nonlinear and hysteretic behaviour of the device. In addition, a closer look at four response loops with different current levels illustrates that the effective stiffness, denoted by the slope of force-displacement curve, ascends with the adding current. This phenomenon is called strain stiffening. When the supplying current is 0 , the shear force almost shows the linear relationships with displacement and velocity, respectively. However, with the increase of the current value, the nonlinear behaviour becomes more and more obvious. This result proves that shear modulus and damping property of the device are the functions of current. And this proposed ANFIS model is able to perfectly demonstrate this unique phenomenon.

Fig. 6 shows the time-series comparison between measured force responses and predictions from the trained ANFIS model when the device is loaded with random excitations. The results again verify that the proposed model can effectively generate the outputs, which meet with the practical data very 
well. Although some deviations exist in the peak regions, ANFIS shows satisfactory performance in prediction of shear force as a whole. Fig. 7 reflects the approximation capacity between experimental and predicted data for both training and validation cases, respectively. The results in the figure shows the data points are mainly distributed in the area of line $y=x$, which indirectly demonstrates that the trained model is qualified for characterize this new device.

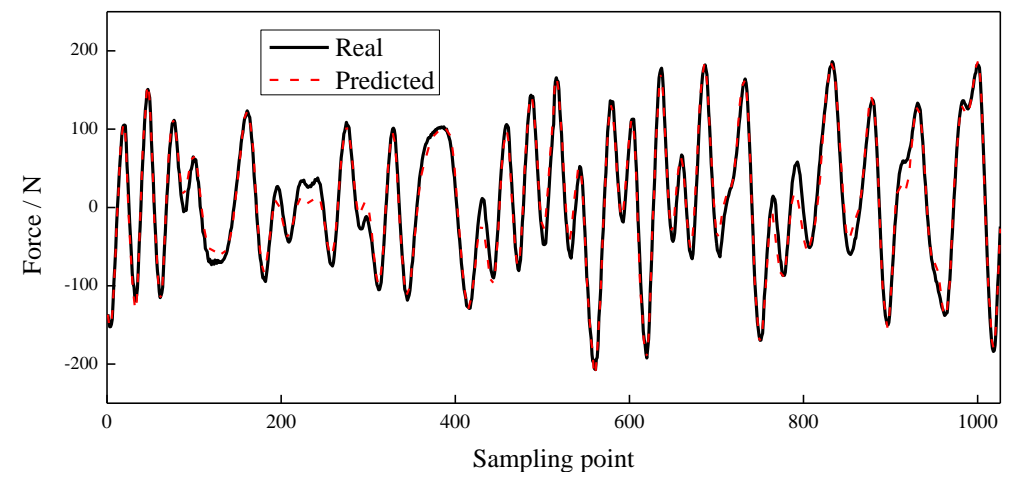

Fig. 6 Time-series comparison between real data and predicted data from the ANFIS model

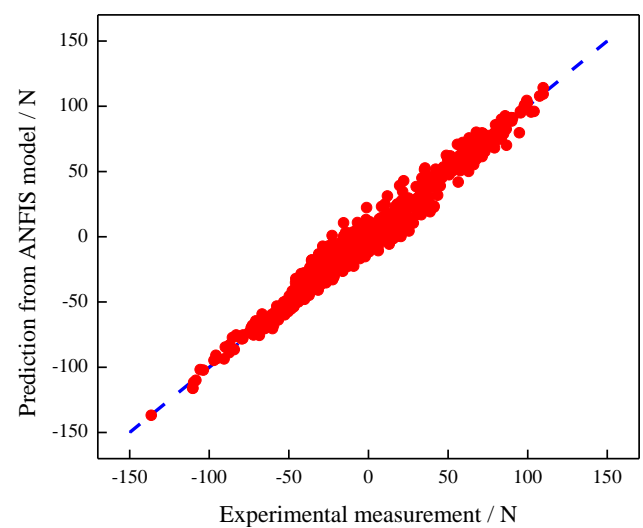

(a) Training samples

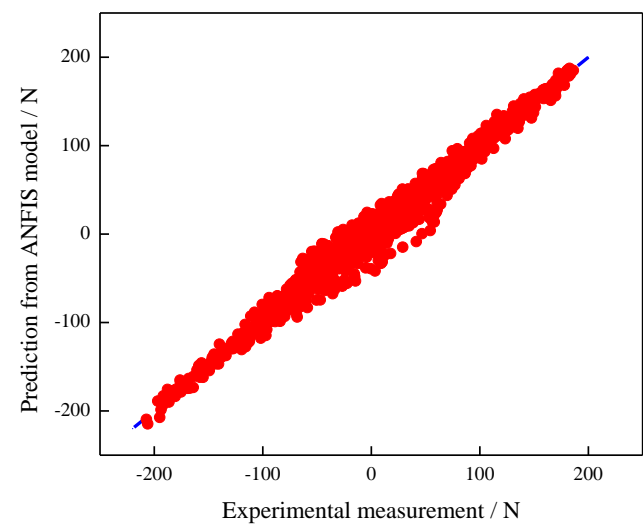

(b) Validation samples

Fig. 7 Result regression analysis of random excitation case

Table 1 Performance comparison among four device models

\begin{tabular}{ccccccccc}
\hline \multirow{2}{*}{ Index } & \multicolumn{2}{c}{ ANFIS } & \multicolumn{2}{c}{ ANN } & \multicolumn{2}{c}{ SVR } & \multicolumn{2}{c}{ BW } \\
\cline { 2 - 10 } & Harmonic & Random & Harmonic & Random & Harmonic & Random & Harmonic & Random \\
\hline MAE & 1.0917 & 1.2535 & 1.1459 & 1.2731 & 1.1386 & 1.2893 & 1.5478 & 1.7342 \\
\hline MRE & $12.11 \%$ & $14.73 \%$ & $13.52 \%$ & $14.97 \%$ & $13.56 \%$ & $15.04 \%$ & $15.92 \%$ & $18.63 \%$ \\
\hline RMSE & 1.5479 & 1.8736 & 1.6528 & 1.9362 & 1.6509 & 1.9477 & 1.8601 & 2.2739 \\
\hline TIC & 0.0006 & 0.0008 & 0.0007 & 0.0008 & 0.0007 & 0.0008 & 0.0009 & 0.0011 \\
\hline
\end{tabular}

To further illustrate the proposed ANFIS superior to other existing models, a comparative study is conducted in this part. The adopted models include artificial neural network (ANN) [10], support vector machine (SVM) [11] and classical Bouc-Wen (BW) model. Besides, mean absolute error (MAE), mean relative error (MRE), root mean square error (RMSE) and Theil inequality coefficient (TIC) are employed for model performance evaluation, where MAE, MRE and RMSE denote forecast accuracy of the ANFIS model and TIC describes the degree of agreement between the ANFIS model and the studied process [12]. Table 1 gives the comparison results of different models based on the validation data of harmonic and random excitation cases. In details, the proposed ANFIS model provides MAE of 1.0917, MRE of 12.11\%, RMSE of 1.5479 and TIC of 0.0006 , which performs better than other three conventional models. As a consequence, the proposed method is able to generate wonderful shear force forecast accuracy for MRE base isolator. 


\section{Summary}

Magnetorheological elastomer (MRE) base isolator is a semi-active device which has obtained more and more attentions in the last few years. The main benefit of this device is that its shear modulus and damping properties will change with the external applied currents. However, the nonlinear force-displacement response of this device is considered as the main challenge for its control application in civil structures. Aiming at this issue, a nonparametric model based on adaptive neuro-fuzzy inference system (ANFIS) is developed to describe the nonlinear and hysteretic behaviours of the MRE base isolator. Finally, experimental data measured at different loading conditions are used to train and validate the proposed ANFIS model. Finally, through comparison with other classical models, the ANFIS method is able to provide more satisfactory results in the indices of MAE, MRE, RMSE and TIC.

\section{References}

[1] K.M. Popp, M. Kroger, W.H. Li, X.Z. Zhang, P.B. Kosasih, MRE properties under shear and squeeze modes and applications,

[2] Y. Yu, Y. Li, J. Li, Parameter identification of an improved Dahl model for magnetorheological elastomer base isolator based on enhanced genetic algorithm, In: Proceedings of the 23rd Australasian Conference on the Mechanics of Structures and Materials. Byron Bay, Australia; 2014, pp. 931-936.

[3] J. Yang, H. Du, W. Li, Y. Li, J. Li, S. Sun, H. Deng, Experimental study and modeling of a novel magnetorheological elastomer isolator, Smart Mater. Struct. 22 (2013) 117001.

[4] Y. Yu, Y. Li, J. Li, A new hysteretic model for magnetorheological elastomer base isolator and parameter identification based on modified artificial fish swarm algorithm, In: Proceedings of 31st International Symposium on Automation and Robotics in Construction and Mining. Sydney, Australia; 2014, pp. 176-183.

[5] Y. Yu, Y. Li, J. Li, Parameter identification and sensitivity analysis of an improved LuGre friction model for magnetorheological elastomer base isolator, Meccanica doi: 10.1007/s11012-015-0179-z.

[6] Y. Li, J. Li, A highly adjustable base isolator utilizing magneotorheological elastomer: experimental testing and modeling, J. Vib. Acoust. 137 (2015) 011009.

[7] Y. Yu, Y. Li, J. Li, Parameter identification of a novel strain stiffening model for magnetorheological elastomer base isolator utilizing enhanced particle swarm optimization, $\mathrm{J}$ Intel. Mat. Syst. Str. doi: 10.1177/1045389X14556166.

[8] Y. Li, J. Li, T. Tian, W. Li, A highly adjustable magnetorheological elastomer base isolator for applications of real-time adaptive control, Struct. 22 (2013) 095020.

[9] J. Yen and R. Langari, Fuzzy Logic: Intelligence Control, and Information. New York, NY: Prentice Hall, 1999.

[10] Y. Yu, Y. Li, J. Li, Nonparametric modeling of magnetorheological elastomer base isolator based on artificial neural network optimized by ant colony algorithm, J Intel. Mat. Syst. Str. 26 (2015) 1789-1798.

[11] Y. Yu, Y. Li, J. Li, Forecasting hysteresis behaviours of magnetorheological elastomer base isolator utilizing a hybrid model based on support vector regression and improved particle swarm optimization, Struct. 22 (2013) 035005.

[12] D. Murray_smith, Methods for the external validation of continuous system simulation models: a review, Math. Comput. Model. Dyn. Syst. 4 (1998) 5-31. 